A N N A L E S Annales de Bretagne et des Pays de l'Ouest

\title{
Le petit faux saunage au XVIIIe siècle en Touraine méridionale
}

Rolande Collas

\section{(2) OpenEdition}

\section{Journals}

Édition électronique

URL : http://journals.openedition.org/abpo/1605

DOI : $10.4000 / a b p o .1605$

ISBN : 978-2-7535-1486-7

ISSN : 2108-6443

Éditeur

Presses universitaires de Rennes

Édition imprimée

Date de publication : 20 juin 2002

Pagination : $59-67$

ISBN : 978-2-86847-742-2

ISSN : 0399-0826

\section{Référence électronique}

Rolande Collas, "Le petit faux saunage au XVIIIe siècle en Touraine méridionale », Annales de Bretagne et des Pays de l'Ouest [En ligne], 109-2 | 2002, mis en ligne le 20 juin 2004, consulté le 01 mai 2019. URL : http://journals.openedition.org/abpo/1605 ; DOI : 10.4000/abpo.1605 


\title{
Le petit faux saunage au XVIII siècle en Touraine méridionale
}

\author{
Rolande COLLAS \\ CEHvi-Université de Tours
}

Si l'on connaît bien désormais les pratiques au XVIII ${ }^{\mathrm{e}}$ siècle de la fraude massive sur le sel et ses acteurs dans l'Ouest de la France, en revanche le petit faux saunage et ses conséquences comme les perquisitions des gabelous chez les petites gens, n'ont pas vraiment retenu l'attention ${ }^{1}$. Pour cette raison l'étude du faux saunage et de ses auteurs dans le ressort des greniers à sel de Preuilly et de La Haye nous a paru intéressante à faire; elle permet aussi de découvrir les méthodes qu'utilisaient les employés de la Ferme générale lors des fouilles et des visites domiciliaires ainsi que la façon dont les gardes appréhendaient les passants franchissant la barrière du pont de La Haye pour passer de Poitou en Touraine.

La capture du faux sel reposait sur une méthode très simple : l'essentiel des recherches consistait à traquer le faux saunier même si celui-ci ne pouvait être qu'un petit trafiquant. Bien que les règlements fussent stricts, les poursuites dans la recherche du faux sel allaient souvent au-delà de ce qui était prévu par la Grande Ordonnance de $1680^{2}$, au sens où les gabelous semblent n'avoir respecté ni les lieux qu'ils inspectaient, ni les demeures qu'ils visitaient, ni parfois les personnes qu'ils fouillaient.

\section{La chasse au petit trafic de faux sel}

Dans un fichier total comprenant 1305 procès-verbaux, nous avons recensé, entre 1700 et 1790 , pour le ressort des greniers à sel de Preuilly et de La Haye l'arrestation de 606 trafiquants de sel dont 148 porte-col, près de 24,5\%, 47 avec des chevaux ou des bêtes azines, 8 avec équipage, 3 en voiture de poste; pour les autres trafiquants de sel, les procès-verbaux ne mentionnent pas le moyen de transport utilisé. Nous ignorons évidemment les effectifs qui échappaient à la vigilance des gardes. Nous avons recensé

1. Collas, Rolande, Faux saunage et répression dans la Touraine méridionale, 1680-1790, 2 tomes, 1999, Diplôme de Recherches Universitaires préparé à l'Université de Tours sous la direction de Brigitte Maillard.

2. BNF, F 26 179, Ordonnance sur le fait des gabelles, p. 362. 
les faux sauniers qui, tentant de franchir les barrières des ponts et les gués des rivières, fuyaient à la vue des gardes postés en embuscade; dans 32 procès-verbaux les employés des gabelles ont indiqué n'avoir pas pu intervenir parce qu'ils étaient trop éloignés, alors qu'ils voyaient des fraudeurs supposés s'enfuir dans les bois ou traverser la rivière à la nage. Pourtant la Ferme possédait en matière de police une tactique qu'elle jugeait infaillible : celle de contrôler et de tenter de surveiller chaque parcelle des zones frontières sur le territoire des grandes gabelles, grâce à une armée de soldats recrutés et formés à cet effet.

Pour les directions de Tours le dispositif de surveillance était organisé en trois lignes et regroupait, en 1787, 1763 hommes sous les ordres de 21 capitaines généraux ${ }^{3}$. La première ligne, composée en grande partie de gardes à pied sédentaires, pénétrait en profondeur en pays rédimé, c'està-dire en Poitou. La seconde ligne, formée pour l'essentiel de gardes à cheval, entrait elle aussi en pays rédimé. Par la disposition de ses gardes la troisième ligne, plus spécifique à la Touraine, couvrait toute la rivière de la Vienne. Pour le ressort des greniers à sel de La Haye et de Preuilly il en allait différemment dans la mesure où le système défensif délaissait la Vienne pour se concentrer sur les rivières de Creuse et de Claise. De toute évidence c'est sur ces deux rivières qu'étaient postées, pour l'essentiel, les brigades, ce qui leur permettait, le cas échéant, de se servir des barques amarrées le long des berges pour effectuer quelques captures.

Au début du $\mathrm{XVIII}^{\mathrm{e}}$ siècle le système défensif couvrait la rivière de Claise dans sa presque totalité et la rivière de Creuse seulement en son cours inférieur. La zone située au-delà de la Claise se trouvait alors totalement dégarnie, d'où l'importance de la traversée de la Claise pour le trafic du faux sel. Bien que la protection des voies d'eau dût perdurer jusqu'à la Révolution, l'organisation défensive subit, au fil du temps, des modifications : l'accroissement des brigades sur la rivière de Creuse se fit au détriment de la Claise, mais la protection de ces deux rivières s'opéra au préjudice des voies terrestres. Alors qu'en 1708-1712 les effectifs se montaient à 53 hommes et en 1761 à 120, ils atteignaient, à la veille de la Révolution, le chiffre de 267 hommes, dont 31 cavaliers, soit plus du double des effectifs de l'année 1761. Qu'en déduire, sinon que la multiplication des brigades dans les dernières années de l'Ancien Régime indique, peut-être, une prolifération du faux saunage mais certainement aussi une volonté accrue de répression. Par leur situation géographique les rivières de la Creuse et de la Claise jouaient donc, dans le trafic du faux sel et le parcours des faux sauniers, un rôle de tout premier plan.

Travaillant 24 heures sur 24 dans leur corps de garde, au pont de La Haye, les gabelous restaient à l'affût du moindre passant qui empruntait la barrière du pont, persuadés, souvent à juste raison, de trouver sur lui du sel prohibé. Lors des contrôles, les gardes agissaient toujours de la même

3. Huvet-Martinet, Micheline, Gabelous et faux sauniers à la fin de l'Ancien Régime, thèse de $3^{\mathrm{e}}$ cycle, Université de Rennes 2, 1981. 
façon; ils demandaient au suspect s'il n'avait rien " contre les ordres du roi "; quelle que fût la réponse, ils se mettaient aussitôt en devoir de procéder à la fouille du suspect. Lorsqu'il s'agissait d'une femme ou d'une jeune fille certains gardes n'hésitaient pas à faire une fouille plus poussée puisque, parfois, le faux sel se trouvait enfoui dans " des petits sacs attachés autour de la jupe " ou que les dits sacs étaient "fixés autour de la jambe [...] entre ses bas et sa peau ${ }^{4}$ ». Quelquefois une femme était chargée de procéder aux fouilles sur les individus de son sexe : " À la barrière du pont de La Haye s'est présentée une femme pour passer du Poitou en Touraine, l'avons fait entrer dans notre corps de garde et l'avons fait visiter par une femme de la brigade qui nous a fait apercevoir sous ses jupes au-dessus de la ceinture deux poches pleines de sel $^{5}$. " Il est impossible de dire si cette pratique était courante car nous avons trouvé très peu de procès-verbaux mentionnant le recours à une femme pour procéder aux fouilles.

Les interventions des gardes s'effectuaient partout, sur les ponts, sur les routes, dans les chemins, dans les bois; de plus elles étaient permanentes et concernaient tout le monde, les hommes, les femmes, les enfants ainsi que les animaux de transport. Tout cheval, tout mulet, toute bête azine rencontré par les gabelous pouvait faire l'objet d'une perquisition. Il est vrai que les bâts, les bâtines et paniers cachaient parfois en leur fond des pochons de sel. Lorsque la bête ne présentait pas de charges suspectes, les gabelous tournaient autour d'elle, essayant de sentir si l'animal n'était pas " ensaliné ", c'est-à-dire si le pelage n'était pas imprégné de l'odeur et du goût du sel, ce qui aurait confirmé que l'animal avait transporté du faux sel. Parfois les gardes ne trouvaient que des chevaux abandonnés avec des sacs de sel, ou des pochons de sel laissés au bord du chemin ou près d'une rivière; le sel était aussitôt confisqué par les gardes. Sur 404 procès-verbaux de sel saisis, 81 captures, soit $20 \%$, se sont effectuées au bord du chemin, en forêt, le long des rivières; 67 saisies, un peu plus de $16 \%$, ont été réalisées à la barrière du pont de La Haye; 160 captures de faux sel, $40 \%$, ont été faites au domicile des contrevenants. Le ressort des greniers à sel de La Haye et de Preuilly était surtout concerné par le petit faux saunage. Les lieux intéressés par les captures se situaient dans une zone proche des greniers à sel, La Haye était le principal endroit où s'effectuaient les saisies de faux sel.

Outre les brigades dites sédentaires, attachées à des points géographiques fixes, tels les ponts, les gués, les postes de gardes, il existait des brigades ambulantes dont le travail consistait soit à tenir des embuscades, soit à faire des perquisitions dans les maisons d'habitation, pour saisir le sel prohibé. Ces mesures, indispensables si l'on voulait faire respecter la volonté du roi, n'en étaient pas moins humiliantes à l'égard de la population gabellante.

4. Arch. dép. d'Indre-et-Loire, 183 B 4, procès-verbaux du 8 décembre 1725.

5. Ibid., procès-verbal du 28 janvier 1787. 


\section{Où se cache le faux sel?}

Sur 431 procès-verbaux de perquisitions conservés aux Archives départementales d'Indre-et-Loire dans les fonds de justice seigneuriales de la baronnie de La Haye et de Preuilly pour la direction de Tours, le dépouillement de 128 procès-verbaux relatifs aux visites domiciliaires nous a permis de mieux connaître le phénomène des perquisitions à domicile et d'avoir un éclairage sur les comportements des gardes des gabelles : convenables lorsqu'il s'agissait de nobles, de notables, d'officiers supérieurs, mais redoutables lors des visites et des perquisitions chez les petites gens.

Les nobles, les bourgeois et les ecclésiastiques étaient protégés en vertu de l'ordonnance de 1680 complétée par l'arrêt du 13 octobre 1722, qui faisait défense " aux capitaines, archers et gardes des gabelles de faire aucune visite dans les maisons ecclésiastiques, nobles, bourgeois, notables qu'en vertu d'une permission par écrit des officiers de nos greniers ${ }^{6}$ ". Les autres habitants couraient le risque d'une visite impromptue; après être arrivés chez les gens les gardes les sommaient de leur représenter leur sel d'impôt et " billet du grenier ". Que la réponse fût affirmative ou négative, les gardes déclaraient qu'ils entendaient faire visite du domicile. Inspectant, en présence des résidents, chaque recoin de la maison, ouvrant les tiroirs, les coffres et les armoires, ils fouillaient de fond en comble la demeure et ses dépendances, décelant rapidement toutes les cachettes imaginées par les fraudeurs, cachettes qui n'étaient guère difficiles à repérer. Bien que variées elles étaient toujours les mêmes; ainsi en allait-il des pots de grès dans une armoire, flacons de terre dans un coffre ou une huche. Quelquefois le pot de terre ou de grès se trouvait dissimulé "dans le four de la boulangerie ${ }^{7}$ ", ou " dans la paillasse du lit dans un petit sac de toile ${ }^{8}$ ", ou encore " dans une fenêtre à moitié masquée par le chevet du lit ${ }^{9}$ ". Parfois, certains particuliers se montraient plus astucieux et cachaient le sel dans des endroits vraiment inattendus : " [...] Faisant nos recherches aurions trouvé sous une ruche à abeilles à côté de la porte une terre nouvellement remuée avec plusieurs pierres qui la couvraient. Nous aurions trouvé à cet endroit une cruche de terre bouchée de mauvais linge. Après l'avoir ouverte avons remarqué que lad. cruche était à mi-pleine de sel qui nous a paru faux ${ }^{10}$. " Une analyse plus fine des procès-verbaux montre qu'il y a, dans le temps, une évolution du type de cache, tant des récipients qui le contenaient que de l'emplacement où l'on dissimulait le faux sel. Ainsi 103 des 128 procès-verbaux indiquent que dans les premières années du siècle le faux sel était pour $14,5 \%$ des cas, dans des pochons, des linges ou des mouchoirs que les gardes trouvaient, le plus souvent, dans les poches des vêtements. Â partir des années 1736 les pots de grès, les bouteilles de

6. BNF, F 46 588, ordonnance sur le fait des gabelles, titre XIX, art. 9, du 13 août 1722.

7. Arch. dép. d'Indre-et-Loire, 183 B 18, procès-verbal du 5 février 1740.

8. Arch. dép. d'Indre-et-Loire, 183 B 13, procès-verbal du 22 janvier 1768.

9. Arch. dép. d'Indre-et-Loire, 183 B, procès-verbal du 22 mai 1773.

10. Arch. dép. d'Indre-et-Loire, 183 B 6, procès-verbal du 26 septembre 1730. 
terre, les petites tinettes remplacent les pochons; le faux sel mis dans des récipients est alors caché dans une armoire ou une maie fermant à clef, derrière une ruche ou enfoui sous une motte de foin. Il y a, de la part des fraudeurs, une recherche dans la façon de soustraire la précieuse denrée à la vue des gardes.

Malgré ces précautions, peu de caches échappaient à la vigilance des gabelous qui connaissaient parfaitement toutes les ruses et les astuces des fraudeurs. Parfois le comportement même des femmes attirait l'attention des gabelous. Devant l'arrivée inopinée des gardes, les femmes, sans doute prises de panique, avaient des gestes inconsidérés, telle cette habitante de Buxeuil, paroisse de La Haye, qui, au moment où les gardes entreprenaient la perquisition de son domicile " aurait levé de sa poche avec fureur et violence un linge qu'elle aurait voulu jeter dans le feu ${ }^{11}$ "; tout aussi inconséquente fut la femme de Saturnin Riardin, à l'instant où les gardes lui signifièrent qu'ils allaient faire perquisition, ils s'aperçurent que "lad. femme Riardin avait toujours les yeux fixés sur une de ses poches "; y mettant aussitôt la main, les gardes retirèrent un petit sac " qu'elle a voulu jeter dans la cour ${ }^{12}$ ".

Si quelques prises étaient plus importantes, le plus souvent elles n'excédaient guère deux ou trois livres. Il en allait autrement pour le sel des grosses salaisons, c'est-à-dire le sel qui servait à la conservation des viandes et des poissons. Il était strictement défendu sous peine d'amende et de confiscation des marchandises de se servir du sel d'impôt pour saler les chairs. Malgré les nombreuses mises en garde la fraude, en ce domaine, était fréquente. Lors de la perquisition de son domicile, le nommé Périgeon avait présenté trois saloirs remplis de " chairs de cochon ", lesquelles furent suspectées d'être salées avec du sel provenant du Poitou : "Avons visité le saloir et après avoir levé deux cruches avons trouvé dans la troisième qu'elle était salée avec du sel du Poitou ${ }^{13}$. " On peut, ici, se poser la question de savoir comment les gardes discernaient le bon sel, celui du grenier à sel de La Haye, du soi-disant faux sel provenant du Poitou. Le sel du Poitou avait la même consistance et la même couleur que celui de la Touraine : extrait des marais salants de la côte atlantique il était, du fait de son " contact avec les particules d'argiles des oillets, humide et gris ${ }^{14}$ ". Or les salins de la côte atlantique alimentaient en sel autant la Touraine que le Poitou. Sans doute fallait-il regarder de très près ce sel pour y voir une quelconque différence.

À côté des petits trafiquants dont les quantités de faux sel n'excédaient guère quelques livres, il existait le faux saunage organisé dont les grandes quantités de faux sel transportées devaient être dissimulées avant d'être revendues. À cet égard les souterrains-refuges, fort nombreux au sud de la

11. Arch. dép. d'Indre-et-Loire, 183 B 6, procès-verbal du 16 janvier 1731 .

12. Arch. dép. d'Indre-et-Loire, 183 B 18, procès-verbal du 12 mai 1782.

13. Arch. dép. d'Indre-et-Loire, 183 B 6, procès-verbal du 6 janvier 1731.

14. Huvet-Martinet, Micheline, L'aventure du sel, Rennes, Ouest-France, 1998, p. 11. 
Touraine, étaient des endroits propices pour dissimuler le faux sel au regard des gabelous, à condition toutefois que les caches ne fussent pas trop proches des maisons d'habitation, sinon les gardes ne manquaient pas d'y faire une visite. D'un agencement rudimentaire, les souterrains-refuges auraient permis aux faux sauniers de déjouer la surveillance des gardes.

\section{La répression et ses victimes}

Lors de la capture du faux saunier les gardes devaient dresser un procès-verbal; pièce maîtresse du dossier il suivait le contrevenant jusqu'à son jugement. La rédaction des procès-verbaux dénotait, de la part des gardes, une certaine conscience professionnelle : le moindre détail y était minutieusement rapporté. Le faux saunier subissait un véritable interrogatoire : les gardes lui demandaient de décliner son nom, son âge, sa profession, la paroisse où il résidait, s'il était marié, célibataire ou veuf. Ensuite ils demandaient au contrevenant de signer ses déclarations. Dans la majorité des cas les faux sauniers disaient "ne savoir signer "; enfin, c'était la saisie du faux sel et la confiscation du récipient dans lequel se trouvait le sel prohibé. Si la quantité de sel saisi était importante ou si le faux saunier avait indiqué son intention de revendre le sel, les gardes le conduisaient dans les prisons du grenier à sel et ils le remettaient aussitôt entre les mains du geôlier qui l'écrouait. La procédure civile commencée à la suite des saisies domiciliaires se faisait dans un délai court, une à deux semaines après l'ouverture du procès. Elle était relativement simple; si le sel, après examen, était reconnu faux, le prévenu était condamné aux peines prescrites par les règlements, à savoir qu'il devait payer une amende de 100, 200, voire 300 livres. À l'inverse de la procédure civile, la procédure criminelle concernait tous les cas où le prévenu était passible d'une peine afflictive, soit parce que la fraude était importante, soit parce que le prévenu faisait le commerce du faux sel. À de rares exceptions près, les faux sauniers qui séjournaient dans les prisons du grenier à sel en attente de leur jugement étaient ceux qui avaient été arrêtés lors d'une embuscade au passage de la barrière du pont de La Haye ou le long d'une rivière. Sur 362 sanctions indiquées dans les procès-verbaux, 178 concernaient les cas des individus qui attendaient dans les prisons du grenier à sel d'être jugés, un peu plus de $49 \%$, ce qui était beaucoup pour un petit ressort comme celui de La Haye ou de Preuilly.

Les peines prononcées contre les faux sauniers paraissent souvent excessives par rapport aux délits commis. Il y avait une réelle disproportion entre la faute de la fraude, laquelle se résumait, souvent, à une ou deux onces de faux sel, et la rigueur des peines. Dans les pays de grande gabelle comme la Touraine, il existait une échelle des amendes. Avant 1680 un faux saunier à porte-col et sans arme était condamné à 100 livres d'amende; après 1680 ce même faux saunier était condamné à 200 livres. Si le faux saunier conduisait des chevaux l'amende était de 300 livres, et si ce faux saunier disait avoir acheté du sel pour le revendre il était, la première fois, 
condamné à 200 livres d'amende, la seconde fois à 500 livres, à 1000 livres la troisième fois et ainsi à proportion en cas de récidive ${ }^{15}$. Aussi étaient-ils nombreux ceux qui ne pouvaient satisfaire au paiement de l'amende, 300 livres représentant plus d'une année de salaire d'un tisserand ${ }^{16}$. Ces sommes d'argent étaient si importantes qu'un système de conversion des peines pécuniaires en cas de non-paiement fut mis en place. Pour les hommes, le châtiment était de deux sortes : la peine du fouet et les galères. Dans le ressort du grenier à sel de La Haye nous avons relevé dans les procès-verbaux sept cas de conversion de la peine pécuniaire en celle du fouet et quatorze cas en peine des galères. En ce qui concerne les femmes s'adonnant au faux saunage, la législation royale avait prévu, en cas de nonpaiement de l'amende, le bannissement pour une durée de cinq ans " hors du ressort du grenier où elles avaient fait le faux saunage, de celle de leur domicile et de celui de Paris ${ }^{17}$ ". Dans le ressort du grenier à sel de La Haye nous avons dénombré dix cas de bannissement, ce qui est peu, compte tenu du nombre de femmes pratiquant le faux saunage. On peut, ici, se demander si la peine de bannissement était bien effective : si pour les hommes la peine des galères était bien réelle, il semble que pour les femmes, le bannissement ne fut pas toujours infligé. De fait la prison était, pour les femmes, la peine le plus souvent utilisée d'autant que certaines condamnées, notamment les femmes sans profession et les mendiantes, se trouvaient dans un tel état de dénuement qu'elles préféraient demeurer en prison plutôt que d'être à la rue.

Grâce aux mentions portées dans les procès-verbaux, nous avons connaissance, en dépit des lacunes, de la profession de 353 faux sauniers; 167, soit 47 \% d'entre eux, se recrutaient dans les métiers de la terre. Si l'on tient compte des métayers, bien que ce groupe social fût le plus aisé de cette société rurale, nous constatons que presque la moitié des de faux sauniers était constituée des gens de la terre, avec une nette proportion de journaliers et de laboureurs. Cette situation ne correspond pas à la réalité sociale; pour la période étudiée les gens de la terre représentaient plus de 70 \% de la population. Si l'on se réfère à Emmanuel Le Roy Ladurie, sur 28 millions d'habitants que comptait la France en 1789, 22 millions étaient des ruraux ${ }^{18}$. De plus les faux sauniers, dans leur grande majorité, étaient des hommes dans la force de l'âge; passé la cinquantaine les personnes pratiquant la contrebande du sel étaient moins nombreuses. Selon 128 procès-verbaux où l'âge des faux sauniers est indiqué, 97 sont âgés de 18 à 49 ans, soit $75 \%$. Bien que l'échantillon soit assez faible, il est assez représentatif de la composition par âges des individus qui s'adonnaient au trafic du sel. Quant aux femmes qui se livraient à la contrebande du sel,

15. BNF, F 26 179, arrêt du Conseil d'État du roi, 25 juillet, $1^{\text {er }}$ août 1719, p. 362.

16. ZYSBERG, André, Les Galériens, vies et destins de 60000 forçats sur les galères de France, 1680-1790, Paris, 1987, p. 107.

17. Arch. dép. d'Indre-et-Loire, 183 B, procès-verbaux du 11/01/1728.

18. Le Roy LadurIE, Emmanuel, « De la Crise ultime à la vraie croissance, 1680-1789 ", Histoire de la France rurale, Duby, G. et Wallon, A. (dir.), Paris 1975, t. 2, p. 354. 
elles étaient surtout nombreuses en période de crise; elles pratiquaient le faux saunage soit pour leur compte personnel, soit pour celui de leur époux, mais le plus souvent par nécessité.

Les archives judiciaires, toujours riches d'enseignements, nous ont permis de mieux comprendre les méthodes pratiquées au XVIII ${ }^{\mathrm{e}}$ siècle dans le ressort des greniers à sel de La Haye et de Preuilly lors des visites domiciliaires et des perquisitions effectuées à la barrière du pont de La Haye, et d'appréhender la façon dont les gardes faisaient irruption chez les résidents; les procédés employés lors des fouilles illustrent assez bien le comportement des gabelous à l'égard des gens qui, dans la plupart des cas, cherchaient tout juste à cacher leur faute sans manifester beaucoup de culpabilité. La contrebande observée dans le ressort du grenier à sel de La Haye et de Preuilly était surtout un faux saunage de petite envergure qui, parfois, s'effectuait en famille. De fait nous ne connaissons qu'une partie des contrevenants, ceux qui se sont fait prendre. Les autres, ceux qui échappaient à la justice faute d'avoir été arrêtés par les gardes, combien étaient-ils? Quel pourcentage de contrevenants représentaient-ils? Peuton penser que la Ferme a privilégié la lutte contre telle ou telle forme de délit selon les périodes?

Quoi qu'il en soit, cette étude met en évidence les réalités des frontières intérieures, dont l'une des caractéristiques est le nombre des rivières, lesquelles étaient sévèrement gardées par les brigades de la Ferme, ce qui montre l'importance qu'avait, pour la contrebande du sel, le passage des voies d'eau. Néanmoins le faible nombre d'actes de rébellion, au moment de l'arrestation en flagrant délit, ne doit pas faire minimiser l'exaspération et l'hostilité de la population face aux pratiques odieuses des fouilles et des perquisitions; les cahiers de doléances en témoignent qui, en 1789, dénoncent le fait " [...] que les habitants du pays de gabelle n'ont aucune tranquillité dans leurs maisons, les commis font journellement des recherches chez eux, ils sont exposés et forcés d'ouvrir à tout instant leurs meubles ${ }^{19}$ ".

19. Cahiers de doléances de la région Centre, paroisse de La Guerche, éd. Denis Jeanson, t. 2 , p. 240 . 


\section{RESUME}

Cet article présente une étude sur les pratiques du faux saunage entre 1680 et 1790 grâce à l'étude des visites domiciliaires, de la fouille des petits trafiquants de sel et des perquisitions. L'essentiel de la documentation a été fournie par les archives des institutions judiciaires des greniers à sel de Preuilly et de la Haye, conservées aux Archives départementales d'Indre-etLoire. Apparaît ainsi nettement l'existence d'une frontière intérieure qui sépare la Touraine, pays de grande gabelle, et le Poitou, province rédimée, et qui court le long des rivières de la Vienne, de la Creuse et de la Claise, toutes sévèrement gardée par les brigade de la Ferme.

\section{ABSTRACT}

This article introduces a study of the pratices of "faux saunage" between 1680 and 1790, through the analysis of domiciliary visits and the search of both people (salt traffickers) and premises. The main body of the documentation has been supplied by the archives of judiciary institutions of the "greniers à sel" in Preuilly and la Haye, preserved in the archives of Archives Départementales d'Indre-et-Loire. Thus is exhibited an interior limit, separating Touraine, - a country of "grande gabelle" -, and Poitou, - a "province rédimée" -, which runs along rivers Vienne, Creuse and Claise strictly guarded by the brigades of the "Ferme". 
